\title{
Disaster Management through Delay Tolerant Networks
}

\author{
Muhammad Nasar Jamal ${ }^{1}$, Raheel Asif ${ }^{1}$, Fawad Nawaz Khan ${ }^{1}$ \\ and Muhammad Zia ur Rehman ${ }^{2}$ \\ ${ }^{1}$ School of Electrical Engineering and Computer Science (SEECS) National \\ University of Science \& Technology (NUST), H-12, Islamabad, Pakistan \\ ${ }^{2}$ Department of Robotics \& Artificial Intelligence, School of Mechanical \& \\ Manufacturing Engineering (SMME), National University of Sciences \& \\ Technology (NUST), H-12, Islamabad Pakistan \\ Email: \{mjamal, rasif and fkhan\}.msee15seecs@seecs.edu.pk, \\ ziaurrehman@smme.edu.pk
}

\begin{abstract}
Natural disasters take place all over the globe thus results in a lot of destruction and loss. The preexisting telecommunication networks mostly collapse during such conditions and the disaster stricken areas are left with no contact with the outside world. As soon as the crisis occurs the response services come in action but due to unavailability of telecommunication network it becomes very difficult to ensure efficient relief services. As a well-managed recovery operation requires proper contact between different entities of the operation, due to unavailability of network, communication can be made possible with Delay Tolerant Network architecture (DTN). DTN is the opportunistic network designed to operate with no or very less connectivity, without any communication infrastructure that is deployed in extreme environments, this makes it suitable to operate in disaster situations. This paper discusses the DTN architecture and gives a survey that explores DTN with specific applications of Disaster Management and also give insight of the disaster mobility management applications (i.e. DTN applications for Proactive Disaster Management which can predict the mobility pattern of people and response vehicles) which could greatly enhance the disaster relief operation. This paper also highlights the problems in deploying the DTN in challenging environments.
\end{abstract}

Keywords: Critical Response Networks, Delay Tolerant Networks, Reactive and Proactive Disaster Management, Routing in DTN

\section{Introduction}

Natural disasters such as Earthquake, Floods, Tornados, and Hurricanes take place throughout the world and bring about chaos and destruction. With the advancement in the field of science and research some of these calamities can be predicted prior to their happening while some cannot be predicted within time like earth quakes, but still some of the losses could be avoided. Well, predicted or unpredicted, disasters cause too much destruction which often lead to unavailability of communication infrastructure, blocked routes that becomes major hurdle for the Emergency services. A well-managed recovery operation requires effective coordination and communication between different entities involved in the recovery process [1]. Damaged preexisting infrastructure accompanied with power restraints blocks the communication. Then comes the time to find an alternative which is a more robust way of communication that could work in hostile environments. There are different alternatives that can be used such as Mobile Ad-Hoc Networks (MANETs), Wireless Mesh Networks (WMNs), Wireless Sensor Networks (WSNs), Terrestrial Trunked Radio (TETRA), Vehicular Ad-Hoc Networks (VANETs) 
and Delay Tolerant Networks (DTN). This paper focuses on the DTN with specific application in Disaster Management.

\section{Disaster Management}

The phrase Disaster management refers to the response and management of the situation and its recovery in a better coordinated fashion. In short, accurate and timely information of the disaster area to the early responding teams can lead to effective and timely help to the people in need of assistance. Research has shown that a successful disaster management is achieved primarily from the activities of the emergency response teams. For these teams to work in an efficient and timely fashion proper coordination is required within the team and between different teams. Disaster management can be categorized in two categories, one is Reactive Disaster Management and the other is Proactive Disaster Management.

1) Reactive Disaster Management: Reactive disaster management refers to the activities that are done in order to mitigate the effects of the disaster and efforts for the rehabilitation after the disaster has occurred. This includes emergency response services, evacuation and medical services, search and rescue operation for the survivors. Emergency management consists of five different phases i.e. prevention, mitigation, preparedness, response and recovery. With reference to the emergency management model, reactive disaster management refers to the response and recovery phase.

2) Proactive Disaster Management: Proactive disaster management refers to the advanced planning to cope up with disaster and to reduce the hazards and losses before it strikes. With reference to the emergency management model it refers to the first three stages i.e. prevention, mitigation and preparedness. A lot of research is going on in this regard to develop different warning systems such as early warning systems. The work proposed in [1] presents a mobility model that models the movement of the population and relief vehicles in case of a disaster by which the relief services could be made more efficient.

The rest of the paper is organized as follows. Section 2 introduces the Delay Tolerant Networks, Bundle Protocol, Custody transfer and reliability in DTNs and Forwarding techniques in DTN. Section 3 gives a survey of all the work done in the field of disaster management with the help of DTNs. Section 4 provides the detail of the challenges in deploying a DTN in real time and the possible future works.

\section{Delay Tolerant Networks}

The history of DTN trace back to Inter-Planetary Network (IPN) which was launched to connect nodes arbitrarily located on different extraterrestrial planets. Deep-space communication suffer from delays, frequent disruption and high error rate. In such scenarios, traditional protocols fail to provide necessary quality of service because these protocols needs to be delay tolerant. IPN is a special case network named as Intermittently Connected Networks (ICNs). ICNs can be added to either terrestrial Internet or it can be deployed at its edges using gateways. These kinds of networks don't have enough information about network topology and other nodes, hence they work on store-carryforward principle. For this purpose high buffer space is needed because next hop is unknown when node receives data packets and so the receiving node has to store and carry data packets and wait for appropriate next hop to appear. When appropriate next hop is available, packets are forwarded.

As there is no end-to-end path from source to destination at any time so to increase message delivery probability, a technique known as flooding is used. In flooding, multiple copies of message are sent to more than one node. Flooding results in overflow of buffer 
space in nodes and buffer space is critical resource in such networks [2]. DTN enables gateway functionality and works above the protocol stack of ICN. DTN provides storage capacity, replication, forward error correction, parallel forwarding and much more to address communication impairments [2]. DTN architecture use new bundle layer along with other layers of traditional IP to extend encapsulation process, new addressing scheme and high storage capacity to deal with disruption for long time in which traditional TCP connection is dropped [2].

\section{A. Bundle Protocol}

Data can be easily transmitted from source to gateways with the help of traditional Internet protocols. But the question is how to transfer data from one region to another with the help of these gateways? To address this problem, new protocols are needed which must be able to adopt according to variable delays, asymmetrically scattered bandwidth and are not chatty in nature. All internet protocols must be terminated at IPN gateways; data should be handed over to new protocols and encapsulated according to the need for deep-space links [4]. One of the significant problem in such environments is bandwidth mismatch between extra-terrestrial and terrestrial networks. Design of transmission and retransmission timers is also complicated because of bandwidth mismatch along with variable delays. A good approach to be followed in such scenarios is to find already defined protocol suite which resembles to the requirements of IPNs even to a lesser extent and to form a stable IPN backbone this protocol suite must be modified to fulfill deep-space communication requirements [3]. The Bundle protocol developed by IRTF DTNRG is the most popular protocol and it is inspired by SMTP protocol [5]. The base of bundle protocol is to combine data and control signals to form a single entity known as bundle and transmit it over DTN.

\section{B. Custody Transfer and Reliability in DTN:}

In DTNs, TCP like re-transmission is inefficient. Bundle protocol use custody transfer to perform re-transmission functionality. In this process, the bundle which is not received by destination is transferred to node other than the source of the bundle to handle retransmission and the bundle holder is known as custodian. Custody of bundle can also be transferred to other nodes as time passes.

Although custody transfer seems to be a good approach to handle reliability issues but there are several problems associated to this approach. First problem is that some consider it to be a new born concept. Others believe that it provides less reliability as compared to TCP and some argue that it is an optimization to end-to-end reliability. An additional option is used to send acknowledgment to source when bundle is received by destination which is consistent with end-to-end reliability approach [6]. So it ensure reliability on hop-by-hop basis rather than end-to-end basis. The second major weakness is that it does not provide any error detection process or corrupted data rejection. It is worth noting that not all applications require error-detection and applications can provide these features by themselves. Another problem with this approach is that it relies only on the interest of node to accept or reject bundle custody. A congested custodian will not be able to free up space for new bundles. Note that bundle's custody transfer would have never succeeded without chattiness [7].

\section{Forwarding Techniques in DTN}

In this section different forwarding techniques that can be used in the Delay Tolerant Network Architecture are discussed.

1) Opportunistic Forwarding: In opportunistic forwarding technique, bundle is forwarded to first opportunity available but due to random walk, this technique have high end-to-end latencies [19]. 
2) Vector-Based Forwarding Protocols: One kind of vector routing is Flooding-based Vector Routing. In this kind of routing each node periodically compute and store information about its location. It also compute its weighted moving average. When two nodes are able to communicate, they exchange their vectors which contain information about direction and velocity. On the basis of this information, next hop decision is taken. This technique reduces the number of duplication [20].

3) Delegation Forwarding: In this type of forwarding technique, quality and level values are assigned to each node. Initially these values are same. Data can only be forwarded if receiver have higher quality than the sender. If communication is successful, sender increases its level to the quality of receiving node. So higher the node's level, less forwarding by that node and hence less bundle replicas [21].

4) Probabilistic Forwarding: Optimal Probabilistic Forwarding (OPF) is the technique which improve delivery rate for specific number of bundle replicas and operate on the principle of hop-count. It has two major components, one is to have knowledge of network, mobility of node and inter-encounters time. While the other is Probability of direct and indirect delivery by using hop-count and lifetime metrics of bundle [22].

5) Load Balancing-based Forwarding: In networks, some nodes are connected to a large number of nodes while others are connected to few nodes. This distribute data unfairly. In DTNs, failures or losses are because of Cost-utility mismatch, Resource depletion and Attacks targeting nodes. To distribute data fairly, we define two metrics inspired from social sciences. The first metric indicate the likelihood that a contact will be maintained over time and strength is measured by frequency of encounters. This metric is called Perceived Interaction Strength (PIS). The second metric is computed by taking into account that how many times a node is chosen to forward message. Bundles can be exchanged only with those nodes which have higher or equal social status. Those having high status will forward messages faster than those having low status [23].

6) Encounter-based Forwarding: In Encounter-based forwarding techniques, future encounters are predicted by using information about previous encounters. The aim of this technique is to increase delivery rate and decrease resource utilization and overhead. In this method, each node calculates two values in order to predict the future encounters. First value is Encounter Value (EV), which is the encounter rate associated with each node and is calculated separately. The second figure that is computed is the number of encounter in some particular time interval and is known as Current Window Counter (CWC) [24]. Based on the values of EV and CWC the future encounters of a node with a particular node are calculated which can be helpful in better forwarding of the bundles.

7) Resource Allocation-based Forwarding: This technique is used in Resource Allocation Protocol for International DTN routing (RAPID). In this technique, sender save a copy of bundle with itself and transfer it to its destination too. RAPID learn network state information with the help of control plane. The following assumptions are made. First, contacts are for short duration. Second, resources and buffer space is limited. Last, destination have enough buffer space to receive and store all the received bundles. RAPID has following components:

- Which bundles to replicate is determined and decided by selection algorithm.

- The usefulness of bundle with respect to specified metric is estimated by Inference algorithm.

- The data required by inference algorithm propagates through In-band Control channel.

RAPID is the first routing protocol defined for DTN to be realistically implemented and tested [25].

8) Network Coding-based Forwarding: This Network Coding-based Forwarding uses 
the advantage of network coding to reduce number of transmissions and network overhead. Network Coding-based Epidemic Routing protocol combines both network coding efficiency and epidemic routing to achieve good performance [26].

\section{Disaster Management through DTN}

In this section we give a survey of the developments and research done in the field of Disaster management using the DTN architecture. In this paper we have highlighted the work done to provide a communication network for the coordination of the rescue operation teams in order to provide better response to the ones in need of assistance and for better recovery from the crisis i.e. Reactive Disaster Management. We will also highlight some remarkable research that provides Proactive disaster management by providing mobility models which could model the movement of the population and relief vehicles in case of a disaster by which the relief services could be made very efficient [1] [8].

There has been a lot of work done in this regard from proposing new protocols to evaluation of the existing ones and new routing protocols and even new systems designs, which could potentially improve the performance of the network in disaster stricken areas. The contributions in this regard are discussed in this section.

In [9] the authors proposed a DTN forwarding protocol which assigns priorities to the messages and then forward them according to their respective priorities. Handling data according to priority is very advantageous especially for the opportunistic networks where continuous connectivity cannot be maintained, as it will forward critical data before the data with less priority. This fact can be a differentiating factor between life and death in disaster scenarios, as a timely information could save lives. In [10] the authors analyzed performance of different forwarding protocols (Epidemic, PRoPHET, MaxProp and TTR) in disaster scenarios. Their analysis showed that MaxProp has the best delivery ratio, while TTR showed best results in case of cost and overhead. Hence it showed that there was no universal best forwarding protocol which could work in every scenario but it depends upon the situation and scenario in which the network is deployed.

In disaster scenarios the routing and forwarding of the disaster data is very critical and that's why a lot of research is going on in this regard. In another such attempt presented in [11] the authors evaluated the performance of the forwarding protocols (Direct delivery, First contact, Epidemic, Spray and wait, Binary spray and wait and PROPHET) in terms of fairness in the delivery of messages between nodes and simulation results showed that none of these forwarding protocols satisfied the fairness criteria. In [12] two new energy efficient forwarding protocols (PropTTR and PropNTTR) were proposed. The energy efficiency is a very crucial element of disaster response networks, as in such conditions it is not possible to charge or change batteries of the deployed nodes hence it is very important that the nodes only consume energy to send critical data and in the meanwhile save energy. Forwarding protocol that needs less number of retransmissions could effectively reduce the energy consumption of the nodes and prolong their lives.

In [13] the authors proposed Enhanced Media Coordinate system for DTN that realize dynamic control of the parameters by the nodes environment, user policies and network conditions in situation of emergencies. The proposed system architecture has three additional functionalities that are Data triage by user specific policies, selection of nodes by its territories and dynamic FEC by population estimation method. Data triage method re shuffles the data stored in the buffer according to the marked importance. Unlike the previous forwarding approaches used by DTN in which the latest data arrives and gets forwarded first and with this the data arrived earlier could have to wait for quite long time [13], which could be very bad for the disaster scenarios in which non important data could be using the scarce network resources and the data important for the relief and recovery operation would be delayed unnecessarily. With the data triage, the proper shuffling of the data before forwarding can ignore the less important data based by the policies of the 
users and give priority to the important data. The idea of data triage is shown in Figure (1).

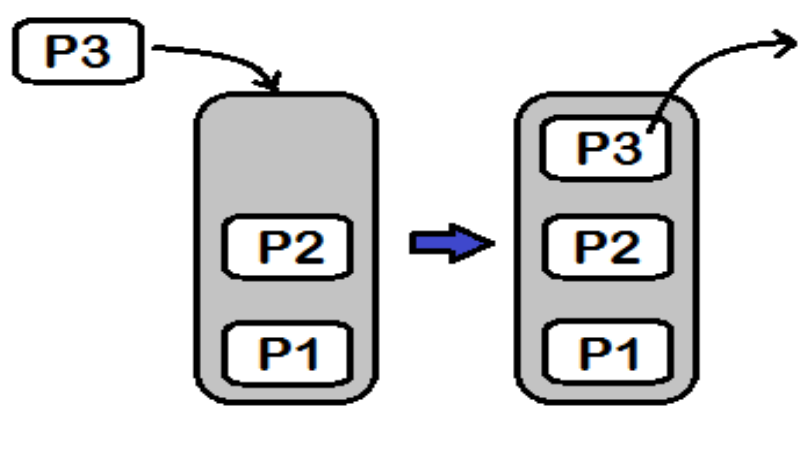

\section{Current DTN}

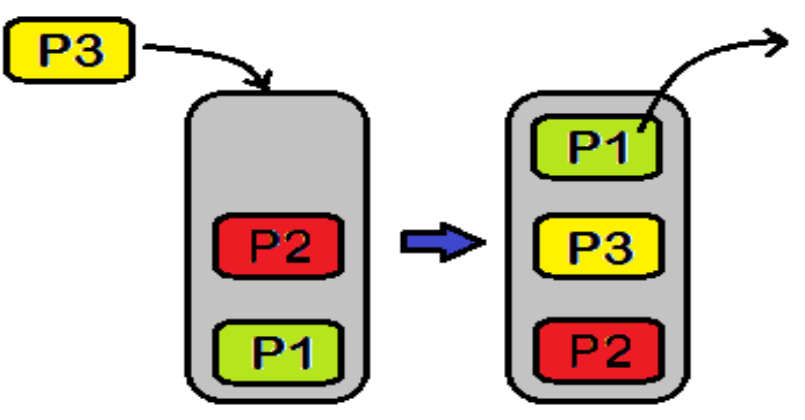

\section{Data Traige}

Figure 1. Difference b/w Data Triage and Normal DTN Forwarding

In [14] Autonomous Flight Wireless Nodes (AFW) based on autonomous flights and data deliveries using the Data Triage based DTN architecture is introduced. During the autonomous flight AFW seeks for radio nodes to deliver data. When AFW detects any radio source it tries to find three location points. Then it finds the middle point of the location by using the formula (1) [14].

$$
\left|\begin{array}{cccc}
x^{2}+y^{2} & x & y & 1 \\
x_{1}^{2}+y_{1}^{2} & x_{1} & y_{1} & 1 \\
x_{2}^{2}+y_{2}^{2} & x_{2} & y_{2} & 1 \\
x_{3}^{2}+y_{3}^{2} & x_{3} & y_{3} & 1
\end{array}\right|=0
$$

When the middle point of the location is calculated the AFW moves to the center of the location and hovers around until the data transmission. After the transmission is complete, it again switches to the autonomous mode and looks for other radio transmission. This system can be very helpful in disaster scenarios for better and timely communication of disaster data which could lead in better response and recovery.

DTN MapEx [15] is a distributed computing system that generates map for the disaster area over the DTN architecture. The volunteers move through their assigned areas and act as the DTN nodes and collect assessment and triage data. These nodes send their data over the DTN to the pre-selected stationary computing nodes, the computing nodes after 
receiving the data compute a digital map of the disaster area using distributed computing and the generated disaster map is routed back to the nodes. After generation of the map of the disaster area, critical areas can be pointed out and the rescue services can be efficiently coordinated and the response and recovery process can be made more efficient.

In [16] the authors propose DistressNet, which is a sensor network architecture which support disaster response. DistressNet uses ad-hoc wireless network architecture and the data communications are enabled with the DTN protocols. It is ad-hoc wireless network architecture with distributive collaborative sensing and topology aware forwarding with accurate resource utilization which supports disaster response. The use of distributed sensing minimizes the energy consumption of the DTN nodes which is a key requirement of the opportunistic networking.

In [17] DTN with CWN (Cognitive Wireless Network) for the disaster communication network has been proposed, consisting of multiple wireless interfaces which could improve the QoS (Quality of Service) of the DTN. In the proposed architecture the fixed and mobile nodes have multiple heterogeneous wireless interfaces such as IEEE802.11a/b/g/n, amateur radio, FWA. The wireless interfaces to be used are selected on the basis of the network conditions, policies, message priorities and nodes movements, to improve the delivery rate and latency and in turn improving the QoS of the DTN.

In [8] the authors proposed a location based mobility prediction scheme using DTN architecture which could aid in the selection of an appropriate forwarding node by predicting the mobility pattern of the forwarding node to increase the reliability and performance of the DTN. In this approach the periodicity of the mobility of the DTN nodes is approximated and this mobility information is used in the selection of the next node to which the data is forwarded. In this approach each mobile node samples its location and this data is exchanged with other nodes upon contact, the location information received from the nodes is analyzed and extrapolated to predict the possible location of the nodes to be in the future for the next few points, the node which is predicted to have the minimum distance around the vicinity of the destination is the selected forwarding node. This could increase the performance of the DTN.

In [1] a post disaster mobility model is proposed which can model the impact of the disaster on the transportation network and models the movement of the population and relief vehicles in case of a disaster. The model has been implemented on Opportunistic Network Environment (ONE) simulator. In this model a pre warned population moves to safety before a disaster scenario occurs, the model predicts the mobility pattern of people and mimics the disaster scenario. With this mobility model the rescue teams can have a pre devised plan of action in case of a disaster and the rescue operations and the recovery can be done more efficiently.

\section{Challenges and Future Works}

1) Routing has been the mostly debated issue of the DTN. There is no routing protocol that is best suited for every scenario as different routing protocols give different performance depending on several conditions. None of the routing protocols proposed for the DTN architecture gives a clear cut procedure for devising path between the nodes. Also all the efforts have been done for the forwarding issues (data plane) and not much work has been done regarding the control plane problems [18].

2) The design of more intelligent, less chatting and efficient network learning procedures is an open research problem [18]. The contact of DTN nodes may either be highly deterministic or totally unpredictable and when there is less network information available then the learning procedures exchange too much data which wastes a lot of bandwidth. 
3) DTN characteristic vary from one environment to another hence developing a generalized DTN model for every network environment is challenging problem in the research community [18].

4) The moment when a bundle reaches its destination, the remaining copies of the bundles are no longer needed and need to be discarded to decrease the forwarding overhead of the nodes. But instructing the other nodes to discard such packet involves further usage of the network resources and is not efficient. This is another problem that needs to be resolved [4] [18].

5) The security of the bundle is still an issue to be resolved as its still in early stages and no proper security protocols have been devised.

6) Whenever the node becomes congested it drops the incoming bundles when overwhelmed. An intelligent way is to inform the node about the probability $\mathrm{p}$ with which the bundle is expected to reach the destination, and below a certain bandwidth occupancy threshold $\beta$, the node accepts the incoming bundles [18]. The studies which determine the optimal value of the $\beta$ and $p$ are a topic of the future studies.

7) As DTN nodes have contact for very short intervals and the inter contact time may be very large, some of the bundle stored in the buffers may not have enough lifetime and could expire before any contact occurs. Discarding these bundles early may reduce the buffer occupancy and might appear as good congestion control mechanism. Future studies might take this in account for further research.

\section{Conclusion}

Whenever a large scale disaster occurs, lack of connection or congestion in the network might cause no or very less information available about effected area and people safety, which is an important factor for an efficient and well-coordinated rescue and recovery operation. In these scenarios DTN is one of the critical response network approach that can be deployed as a Disaster Information Network system, which can aid in efficient Disaster Management. It is seen from the survey given in this paper that DTN is very efficient approach to be used in the reactive disaster management. As the Disaster Response Network created by DTN can make the initial response process very efficient by enabling communication between different entities of the response process and thus timely help can be provided to people in need of assistance. DTN has also some applications in proactive disaster management such as predicting the mobility models of the people and response vehicle if a disaster may happen. With this, the response process can be pre managed and efficiently employed. However, there are some difficulties in the deployment of DTN as discussed and are under research. With these issues being catered for, DTN can prove to be a very efficient Disaster Management tool which can make a great difference in life and death situations.

\section{References}

[1] Uddin, Md Yusuf S., David M. Nicol, Tarek F. Abdelzaher, and Robin H. Kravets. "A post-disaster mobility model for delay tolerant networking." InWinter Simulation Conference, pp. 2785-2796. Winter Simulation Conference, 2009.

[2] M. Khabbaz, C. Assi and W. Fawaz, "Disruption-Tolerant Networking: A Comprehensive Survey on Recent Developments and Persisting Challenges," IEEE Communication Surveys and Tutorials 14(2), 607-640 (2012).

[3] V. Cerf, S. Burleigh, A. Hooke, L. Torgerson, R. Durst, K. Scott, E. Travis and H. Weiss, "Interplanetary Internet (IPN): Architectural Definition," Available Online: http://www.ipnsig.org/reports/ memo-ipnrg-arch-00.pdf.

[4] Fall, Kevin. "A delay-tolerant network architecture for challenged internets." In Proceedings of the 2003 conference on Applications, technologies, architectures, and protocols for computer communications, 
pp. 27-34. ACM, 2003.

[5] Scott, K., and S. Burleigh. "Bundle Protocol Specification, IETF RFC 5050, Experimental." (2007).

[6] Farrell, Stephen, and Vinny Cahill. Delay-and disruption-tolerant networking. Artech House, Inc., 2006.

[7] Wood, Lloyd, Wesley M. Eddy, and Peter Holliday. "A bundle of problems." In 2009 IEEE Aerospace conference, pp. 1-17. IEEE, 2009.

[8] Ganguly, Sandipan, Souvik Basu, Siuli Roy, and Suvankar Mitra. "A location based mobility prediction scheme for post disaster communication network using DTN." In Applications and Innovations in Mobile Computing (AIMoC), 2015, pp. 25-28. IEEE, 2015.

[9] Joe, Inwhee, and Sang-Bo Kim. "A message priority routing protocol for delay tolerant networks (DTN) in disaster areas." In International Conference on Future Generation Information Technology, pp. 727737. Springer Berlin Heidelberg, 2010.

[10] MartîN-Campillo, Abraham, Jon Crowcroft, Eiko Yoneki, and Ramon Martí. "Evaluating opportunistic networks in disaster scenarios." Journal of Network and Computer Applications 36, no. 2 (2013): 870880.

[11] Takahashi, Asato, Hiraki Nishiyama, and Nei Kato. "Fairness issue in message delivery in delay-and disruption-tolerant networks for disaster areas." In Computing, Networking and Communications (ICNC), 2013 International Conference on, pp. 890-894.IEEE, 2013.

[12] Martín-Campillo, Abraham, and Ramon Martí. "Energy-efficient forwarding mechanism for wireless opportunistic networks in emergency scenarios."Computer Communications 35, no. 14 (2012): 17151724.

[13] Uchida, Noriki, Noritaka Kawamura, Kazuo Takahata, Yoshitaka Shibata, and Norio Shiratori. "Proposal of data triage methods for disaster information network system based on delay tolerant networking." In Broadband and Wireless Computing, Communication and Applications (BWCCA), 2013 Eighth International Conference on, pp. 15-21. IEEE, 2013.

[14] Uchida, Noriki, Noritaka Kawamura, Tomoyuki Ishida, and Yoshitaka Shibata. "Proposal of Autonomous Flight Wireless Nodes with Delay Tolerant Networks for Disaster Use." In Innovative Mobile and Internet Services in Ubiquitous Computing (IMIS), 2014 Eighth International Conference on, pp. 146-151. IEEE, 2014.

[15] Trono, Edgar Marko, Yutaka Arakawa, Morihiko Tamai, and Keiichi Yasumoto. "DTN MapEx: Disaster area mapping through distributed computing over a Delay Tolerant Network." In Mobile Computing and Ubiquitous Networking (ICMU), 2015 Eighth International Conference on, pp. 179-184. IEEE, 2015.

[16] George, Stephen M., Wei Zhou, Harshavardhan Chenji, Myounggyu Won, Yong Oh Lee, Andria Pazarloglou, Radu Stoleru, and Prabir Barooah. "DistressNet: a wireless ad hoc and sensor network architecture for situation management in disaster response." Communications Magazine, IEEE 48, no. 3 (2010): 128-136.

[17] Uchida, Noriki, Noritaka Kawamura, Nicholas Williams, Kazuo Takahata, and Yoshitaka Shibata. "Proposal of delay tolerant network with cognitive wireless network for disaster information network system." In Advanced Information Networking and Applications Workshops (WAINA), 2013 27th International Conference on, pp. 249-254 IEEE, 2013.

[18] Khabbaz, Maurice J., Chadi M. Assi, and Wissam F. Fawaz. "Disruption-tolerant networking: A comprehensive survey on recent developments and persisting challenges." IEEE Communications Surveys \& Tutorials 14, no. 2 (2012): 607-640.

[19] P. Basu and C. K. Chau, "Opportunistic Forwarding in Wireless Networks with Duty Cycling," Proc. ACM MobiCom Workshop on Challenged Networks, San Fransisco, C.A., September 2008.

[20] H. Kang and D. Kim, "Vector Routing for Delay Tolerant Networks," IEEE Vehicular Technology Conference, Calgary, B.C., Canada, pp. 15, September 2008.

[21] V. Erramilli, M. Crovella, A. Chaintreau and C. Diot, "Delegation Forwarding," Proc. ACM MobiHoc, pp. 251-259, May 2008.

[22] C. Liu and J. Wu, "An Optimal Probabilistic Forwarding Protocol in Delay Tolerant Networks," Proc. ACM MobiHoc, New Orleans, Louisiana, United States, May 2009.

[23] J. M. Pujol, A. L. Toledo and P. Rodriguez, "Fair Routing in Delay Tolerant Networks," Proc. IEEE INFOCOM, Rio De Janeiro, Brazil, April 2009.

[24] S. C. Nelson, M. Bakht and R. Kravets, "Encounter-Based Routing in DTNs," Proc. IEEE INFOCOM, Rio De Janeiro, Brazil, April 2009.

[25] Balasubramanian, B. N. Levine and A. Venkataramani, "DTN Routing as a Resource Allocation Problem," Proc. ACM SIGCOMM, Kyoto, Japan, August 2007.

[26] Y. Lin, B. Liang and B. Li, "Performance Modeling of Network Coding in Epidemic Routing," Proc. ACM MobiOpp, San Juan, Puerto Rico, United States, June 2007. 
International Journal of Future Generation Communication and Networking Vol.10, No.3 (2017) 